\title{
Clinicopathological Features of Autoimmune Hepatitis with IgG4-Positive Plasma Cell Infiltration
}

\author{
Yoshitaka Arase $^{a}$ Koshi Matsumoto ${ }^{b}$ Kazuya Anzai ${ }^{a}$ Kota Tsuruya ${ }^{a}$ \\ Satoru Sugiyama ${ }^{a}$ Shihou Yoshihara $^{a}$ Shunji Hirose $^{a}$ Haruki Uojima $^{c}$ \\ Hisashi Hidakac Takahide Nakazawac Ryuzo Deguchia Seiichiro Kojima ${ }^{\mathrm{a}}$ \\ Shinji Takashimizu ${ }^{a}$ Koichi Shiraishi ${ }^{a}$ Takayuki Shirai $^{a} \quad$ Tatehiro Kagawa $^{a}$
}

aDepartment of Gastroenterology and Hepatology, Tokai University School of Medicine, Isehara, Japan; bDepartment of Pathology, Ebina General Hospital, Ebina, Japan; 'Department of Gastroenterology, Kitasato University School of Medicine, Sagamihara, Japan

\section{Keywords}

Autoimmune hepatitis · Immunoglobulin G4 · Plasma cell · Relapse · Prognosis

\begin{abstract}
Background: We aimed to elucidate the characteristics and prognosis of autoimmune hepatitis $(\mathrm{AIH})$ patients with immunoglobulin (lg) G4-positive plasma cell infiltration. Methods: We enrolled 84 AlH patients. The number of IgGand IgG4-positive plasma cells was immunohistochemically counted per high-power field in the portal area. Patients with 3 or more lgG4-positive plasma cells on average and a ratio of lgG4 to lgG-positive plasma cells $\geq 5 \%$ were defined as IgG4-associated AlH (IgG4-AlH), and their clinicopathological characteristics and prognosis were compared to those of the remaining classical-AlH patients. Results: Ten (11.9\%) and 74 patients (88.1\%) were categorized as IgG4$\mathrm{AlH}$ and classical-AIH patients, respectively. The median age
\end{abstract}

$\begin{array}{ll}\text { karger@karger.com } & \begin{array}{l}\text { (C) } 2020 \text { The Author(s) } \\ \text { Published by S. Karger AG, Basel }\end{array} \\ \text { Www.karger.com/ddi } & \begin{array}{l}\text { This is an Open Access article licensed under the Creative Commons } \\ \text { Attribution-NonCommercial-4.0 International License (CC BY-NC) } \\ \text { (http://www.karger.com/Services/OpenAccessLicense), applicable to } \\ \text { the online version of the article only. Usage and distribution for com- } \\ \text { mercial purposes requires written permission. }\end{array}\end{array}$

of the IgG4-AlH patients was 67 years, the majority was female $(80.0 \%)$, and the distribution was similar to that of the classical-AlH patients. The IgG4-AIH patients exhibited significantly more severe phenotypes in portal inflammation, interface hepatitis, fibrosis, and rosette formation. All clinical laboratory data were similar except for serum lgG4 levels, which were higher in IgG4-AlH patients (168.5 vs. 22.9 $\mathrm{mg} / \mathrm{dL}, p=0.014$ ). During a median follow-up period of 139 months, the relapse rate was significantly lower in the lgG4AlH group than in the classical-AlH group (11.1 vs. $49.2 \%$; $p=0.048)$. Twelve (16.2\%) and $6(8.1 \%)$ classical-AlH patients underwent liver-related events and liver-related deaths, respectively. In contrast, none of the IgG4-AlH patients progressed to severe liver disease. Conclusions: The lgG4-AlH patients had more severe inflammation and advanced fibrosis in the liver. However, their prognosis was not poor compared to that of classical-AIH patients. IgG4AlH may have a phenotype distinct from classical-AlH.

(C) 2020 The Author(s)

Published by S. Karger AG, Basel

Yoshitaka Arase

Gastroenterology and Hepatology, Tokai University School of Medicine

143 Shimokasuya

Isehara 259-1193 (Japan)

ay114535@tsc.u-tokai.ac.jp 


\section{Introduction}

Autoimmune hepatitis ( $\mathrm{AIH})$ is a chronic progressive liver disease characterized by elevated serum alanine aminotransferase (ALT) levels, hypergammaglobulinemia, autoantibodies, and histological features including interface hepatitis, plasma cell infiltration, and rosette formation $[1,2]$. The diagnosis of AIH is made based on the revised International Autoimmune Hepatitis Group (IAIHG) scoring system [3]. The prognosis of AIH is generally good with favorable response to immunosuppressive treatment. However, the prognosis of patients who have persistent elevation of serum ALT levels or who undergo repeated relapses is not necessarily good; they sometimes progress to hepatocellular carcinoma (HCC) or liver failure $[4,5]$.

Recently, a new disease entity called immunoglobulin G4 (IgG4)-associated AIH (IgG4-AIH), which is characterized by the hepatic accumulation of IgG4-bearing plasma cells with markedly elevated serum IgG4 levels in the AIH case, was proposed by Umemura et al. [6]. Although there are a few studies on IgG4-AIH [7-10], the characteristics of this disease remain largely unknown. In particular, the long-term prognosis has not been reported. In this study, we aimed to elucidate the clinicopathological characteristics and clinical course of IgG4-AIH.

\section{Materials and Methods}

\section{Study Design}

This multicenter, retrospective study was conducted at 3 institutions in Japan. The protocol of this study was approved by the Institutional Review Board for Clinical Research of Tokai University School of Medicine (no. 13R-120). All procedures were performed in accordance with the ethical standards of the institutional research committee and with the 1964 Helsinki Declaration and its later amendments or comparable ethical standards. It was judged that written informed consent was not required in the Institutional Review Board for Clinical Research of Tokai University School of Medicine (no. 13R-120) because this research was a retrospective observational study and did not use genetic testing or additional samples from patients. Informed consent was obtained from all the participants by the opt-out method of the poster.

\section{Study Population}

We enrolled 84 patients with biopsy-proven AIH who were followed up for more than 6 months at Tokai University Hospital, Tokai University Hachioji Hospital, and Kitasato University Hospital between October 1993 and December 2012. All patients underwent liver biopsy and were diagnosed as having probable or definite AIH according to the IAIHG scoring system [3]. The following patients were excluded from the study: those with (1) positivity for hepatitis B surface antigen, (2) positivity for antibody to hepatitis $\mathrm{C}$ virus, (3) positivity for an antimitochondrial antibody or M2 antibody, and (4) presence of other liver diseases such as primary biliary cholangitis, primary sclerosing cholangitis, and drug-induced liver injury. All patients were treated based on the Japanese guideline for AIH [11].

\section{Clinical and Laboratory Parameters}

We collected baseline information such as age, gender, concurrent autoimmune diseases, IAIHG score, the presence or absence of liver cirrhosis (LC) at diagnosis, and laboratory parameters including antinuclear antibody (ANA), anti-smooth muscle antibody (ASMA), serum albumin, aspartate aminotransferase, ALT, alkaline phosphatase, total bilirubin, IgG, and IgG4. Serum IgG4 concentrations were measured in 30 cases. A titer of 1:40 or higher was considered positive for ANA and ASMA. Diagnosis of LC was made based on liver histology, computed tomography, ultrasonography, and the presence of esophageal varices and/or nonmalignant ascites.

\section{Histological Evaluation and Immunohistochemistry for IgG} and IgG4, and Definition of IgG4-AIH

Liver biopsy was performed percutaneously with a 16-gauge needle under ultrasound guidance before initiating treatment. The lengths of the specimen were over $15 \mathrm{~mm}$, and each included an average of 13 (range: 6-31) portal tracts. Liver tissues were fixed in formalin and embedded in paraffin, and then $4 \mu \mathrm{m}$ sections were cut out of each paraffin block. Sections were stained with hematoxylin and eosin, Azan-Mallory, and silver impregnation reticulin to evaluate histological features. All liver specimens were blindly assessed by an experienced liver pathologist. Portal inflammation, interface hepatitis, confluent necrosis, and fibrosis were quantitatively evaluated based on the Ishak scoring system [12]. The following histological features were semi-quantitatively categorized as follows: plasma cell infiltration: 0 (absent), 1 (1-4 cells per highpower field [/HPF]), 2 (5-9 cells/HPF), and 3 ( $\geq 10$ cells/HPF); rosette formation: 0 (absent), 1 ( $<3$ cells/HPF), and 2 ( $\geq 3$ cells/HPF); bile duct damage: 0 (absent) and 1 (present); and steatosis: 0 (absent), 1 (fatty change in $<1 / 3$ areas/tissue), 2 (1/3-2/3 areas/tissue), and 3 ( $>2 / 3$ areas/tissue).

Immunostaining for IgG and IgG4 was performed using a rabbit polyclonal antibody against human IgG (DakoCytomation, Glostrup, Denmark) and a mouse monoclonal antibody against human IgG4 (Invitrogen Corporation, Camarillo, CA, USA), respectively, according to the manufacturer's instructions. We counted the number of IgG- and IgG4-positive plasma cells in more than 3 portal areas/HPF and calculated the average number per portal area. We also calculated the ratio of IgG4- to IgG-positive plasma cells (IgG4/IgG ratio) for each case.

We defined IgG4-AIH as patients with 3 or more IgG4-positive plasma cells/HPF on average and an IgG4/IgG ratio of $5 \%$ or higher (Fig. 1a). The other remaining patients were classified as classical-AIH.

\section{Treatment and Follow-Up}

Most AIH patients were treated with prednisolone (PSL) (20$60 \mathrm{mg} /$ day) with or without ursodeoxycholic acid (UDCA) (600 $\mathrm{mg} /$ day), while some patients were treated with UDCA (600 mg/ day) alone because of low-grade activity in liver histology. Japanese AIH patients have better response to corticosteroid treatment than those in Western countries, so the Japanese guidelines indicate 


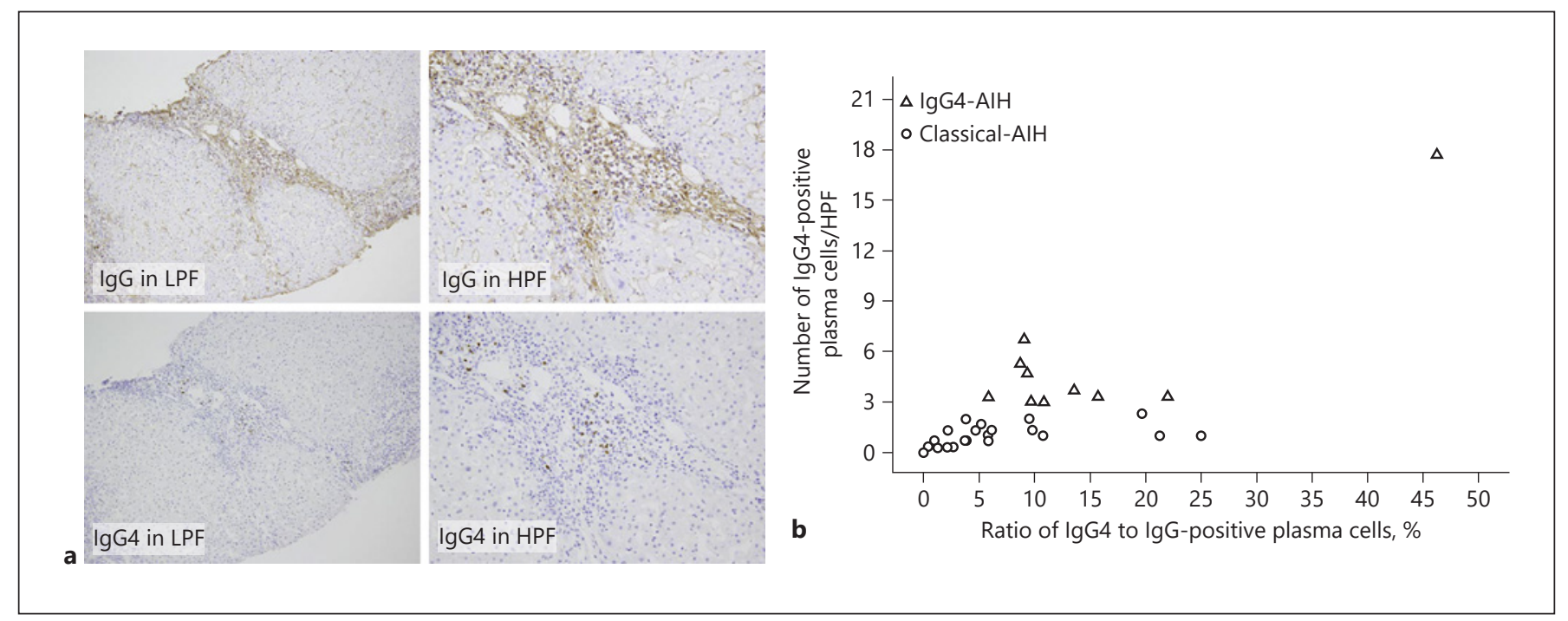

Fig. 1. a Immunohistochemical staining for IgG and IgG4 in the livers of patients with IgG4-AIH: IgG in LPF, IgG in HPF, IgG4 in LPF, and IgG4 in HPF. b Relationship between the number of IgG4-positive plasma cells/ $\mathrm{HPF}$ and the ratio of IgG4 to IgG-positive plasma cells (\%). Ig, immunoglobulin; IgG4-AIH, IgG4-associated autoimmune hepatitis; LPF, low-power field; HPF, high-power field.

that PSL should be administrated at an initial daily dose of $0.6 \mathrm{mg} /$ $\mathrm{kg}$ or more [11]. In addition, the Japanese treatment guideline stated that UDCA $600 \mathrm{mg}$ /day may be administrated concomitantly during the tapering of the PSL dose, or alone in mild cases [11]. After the normalization of serum ALT levels, the PSL dose was tapered by $2.5-5 \mathrm{mg}$ every 1 or 2 weeks to a maintenance dose of 10 $\mathrm{mg} /$ day or less. Relapse was defined as an increase in serum ALT levels above twice the upper limit of normal value (>70 IU/L). When patients relapsed, we increased the PSL dose or initiated azathioprine (50-100 mg/day) as an add-on therapy. If the normalization of serum ALT continued for more than 2 years at the maintenance dose, PSL withdrawal was considered.

Patients were required to visit the outpatient clinic every 2 or 3 months and underwent a physical examination and laboratory blood tests on each visit. Abdominal ultrasonography, computerized tomography, or magnetic resonance imaging was performed every 6 months for evaluation of HCC and ascites. Esophagogastroduodenoscopy was performed every 12 months for evaluation of esophageal varices. Patients were followed up until death or November 30, 2019. The liver-related events were defined as the development of esophageal varices requiring treatment, ascites, and HCC. Liver-related death was defined as death from liver failure or HCC progression.

\section{Statistical Analysis}

Continuous variables were compared between IgG4-AIH and classical-AIH using the Mann-Whitney $U$ test. Categorical variables were compared using the $\chi^{2}$ test or Fisher's exact test where appropriate. The correlations between the numbers of IgG- and IgG4-positive plasma cells in liver tissues, and between serum IgG4 levels and the number of IgG4-positive plasma cells were evaluated using Pearson's rank correlation coefficient. A receiver operating characteristic (ROC) curve was created to obtain the best cutoff value of the number of IgG4-positive plasma cells for the high levels of serum IgG4 concentrations ( $\geq 135 \mathrm{mg} / \mathrm{dL})$. The logrank test using the Kaplan-Meier method was used to compare the rates of liver-related events and liver-related deaths. All analyses were performed using SPSS version 26 (SPSS Japan, Tokyo, Japan), and $p$ values $<0.05$ were considered statistically significant.

\section{Results}

\section{Characteristics of the Study Population}

We enrolled 84 patients: 72 (85.7\%) and 12 (14.3\%) patients were diagnosed as definite and probable AIH according to the IAIHG score (Table 1). Of the 84 patients, $10(11.9 \%)$ and $74(88.1 \%)$ patients were categorized to IgG4- and classical-AIH, respectively (Fig. 1b). The number of IgG4-positive plasma cells and the IgG4/IgG ratio were $5.4 \pm 4.5$ (mean \pm standard deviation [SD]) cells/ $\mathrm{HPF}$ and $15.1 \pm 11.8 \%$, respectively, in the IgG4-AIH group, whereas they were $0.3 \pm 0.6$ cells/HPF and $2.0 \pm$ $4.8 \%$, respectively, in the classical-AIH group. Notably, IgG4-positive plasma cells were not observed in 53 patients $(63.1 \%)$. There were significant positive correlations between the number of IgG- and IgG4-positive plasma cells in the liver tissue (correlation coefficient $=0.352$, $p=0.001$; Fig. $2 \mathrm{a}$ ) and between the serum IgG4 levels and the number of IgG4-positive plasma cells in the liver tissue (correlation coefficient $=0.548, p=0.002$; Fig. 2b). The best cutoff value (Youden index) for the number of IgG4-positive plasma cells using the ROC curve for se- 


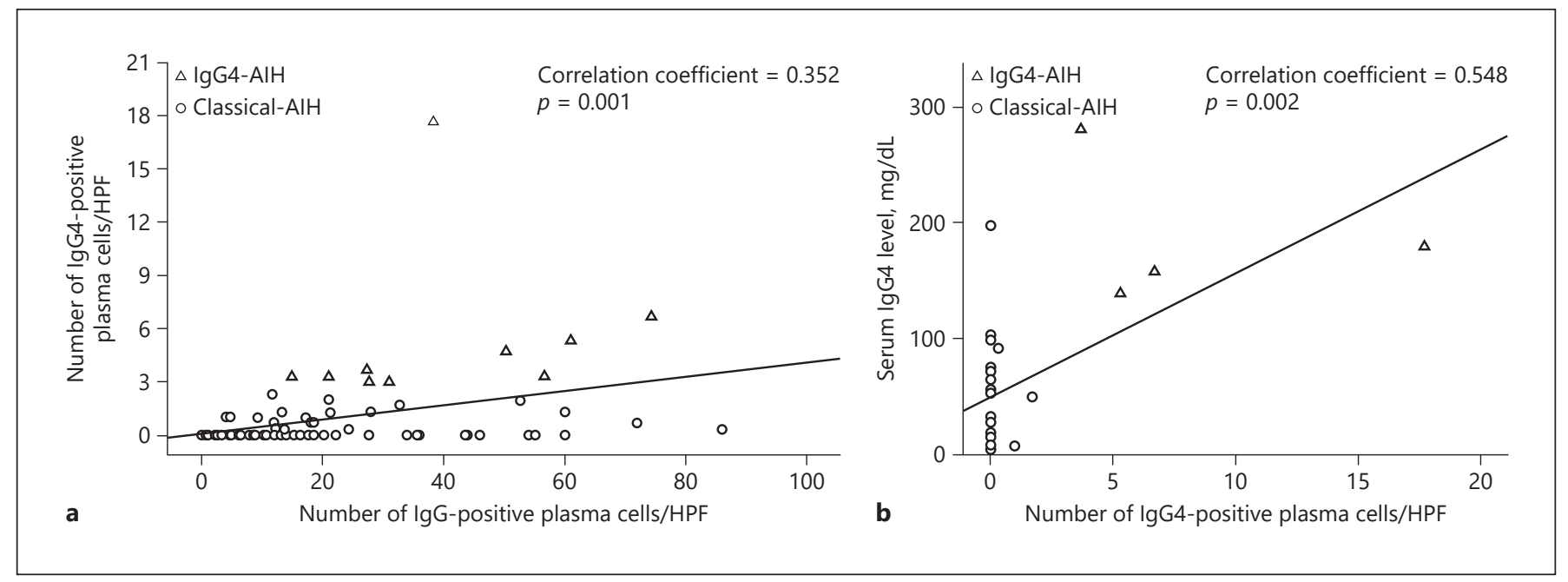

Fig. 2. a Correlations between the number of IgG-positive plasma cells and the number of IgG4-positive plasma cells in the liver tissue (Pearson's rank correlation coefficient). b Correlations between the serum IgG4 levels and the number of IgG4-positive plasma cells in the liver tissue (Pearson's rank correlation coefficient). Ig, immunoglobulin.

Table 1. Clinical characteristics of patients at the time of diagnosis

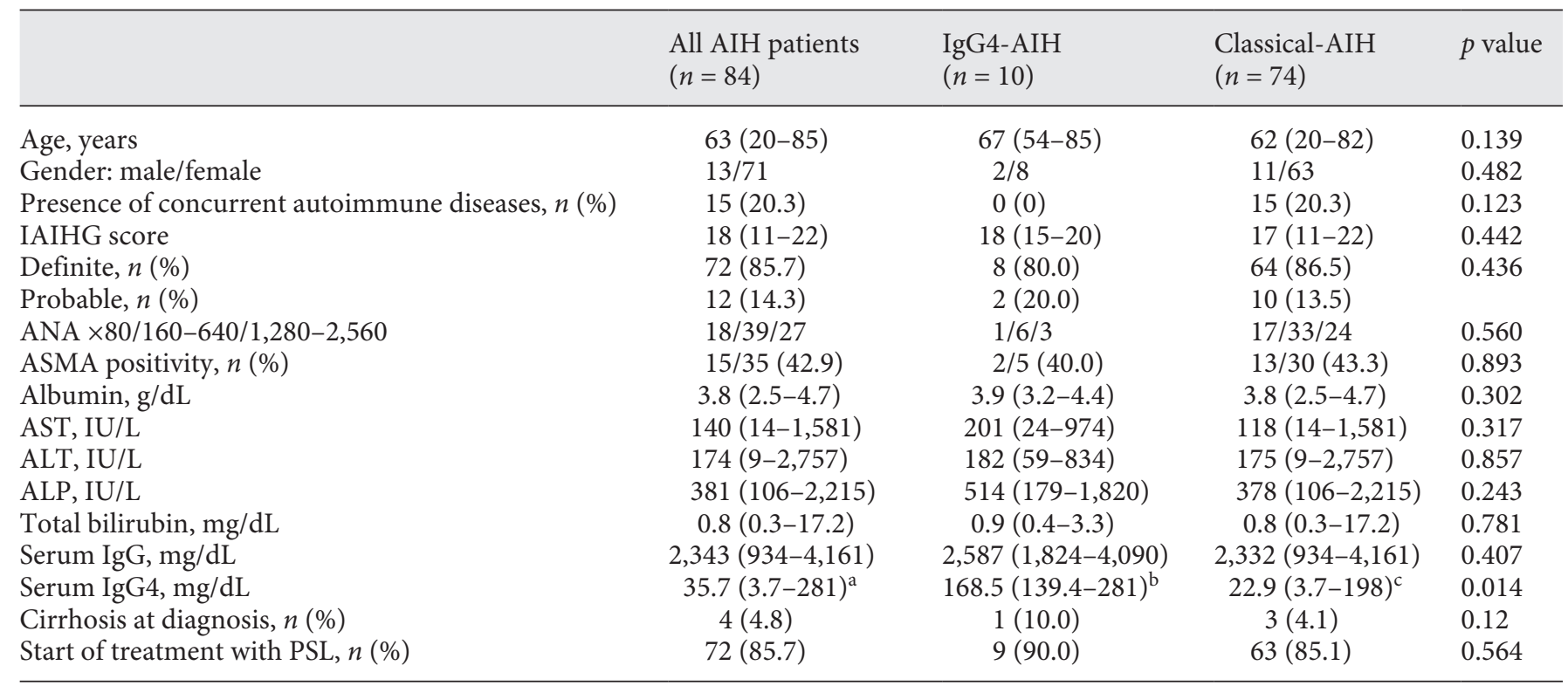

Data are expressed as median (range) or $n(\%)$. AIH, autoimmune hepatitis; IAHG, International Autoimmune Hepatitis Group; ANA, antinuclear antibody; ASMA, anti-smooth muscle antibody; AST, aspartate aminotransferase; ALT, alanine aminotransferase; ALP, alkaline phosphatase; Ig, immunoglobulin; PSL, prednisolone. ${ }^{\mathrm{a}} n=30 .{ }^{\mathrm{b}} n=4 .{ }^{\mathrm{c}} n=26$.

rum IgG4 level $\geq 135 \mathrm{mg} / \mathrm{dL}$ was 2.7 cells/HPF with the area under the curve of 0.89 , sensitivity of $80 \%$, and specificity of $100 \%$. As a result, we adopted 3 or more IgG4positive plasma cells/HPF as part of the definition of IgG4-AIH.

\section{Comparison between IgG4-AIH and Classical-AIH at Baseline}

The median age of the IgG4-AIH patients was 67 (range: 53-85) years, and the majority were female $(80.0 \%)$. This distribution was similar to the classical- 
Fig. 3. Comparison of the histological findings between IgG4-AIH and classical-AIH. The scores of IgG4-AIH patients in portal inflammation, interface hepatitis, fibrosis, and rosette formation were higher than those of classical-AIH patients. Data are shown as mean \pm standard deviation. ${ }^{*} p<$ 0.05. Ig, immunoglobulin; IgG4-AIH, IgG4-associated autoimmune hepatitis; HPF, high-power field.

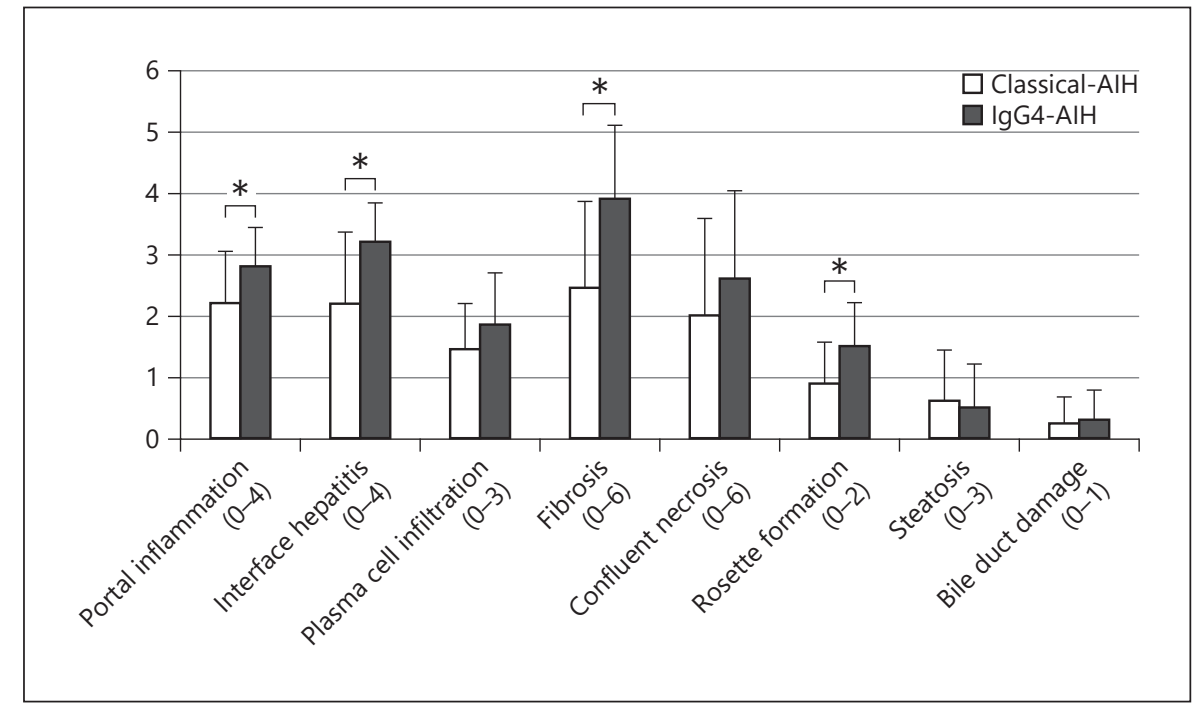

AIH patients (age: 62 [range: $20-82$ ] years, female: $85.1 \%$ ). Fifteen patients $(20.3 \%)$ in the classical-AIH group had concurrent autoimmune diseases (5 with Sjögren's syndrome, 4 with systemic lupus erythematosus, 2 with autoimmune thyroiditis, etc.), whereas the IgG4-AIH patients did not have any concurrent autoimmune diseases $(p=0.123)$.

Clinical laboratory data including serum albumin, aspartate aminotransferase, ALT, alkaline phosphatase, total bilirubin, IgG, the positive rate of ASMA, and the ANA titer were not significantly different between the 2 groups. On the other hand, serum IgG4 levels were significantly higher in the IgG4-AIH $(n=4)$ than the classical-AIH patients $(n=26)$ (median: 168.5 [range: $139.4-$ 281 ] mg/dL vs. median: 22.9 [range: $3.7-198$ ] mg/dL, $p=$ $0.014)$. One IgG4-AIH and 3 classical-AIH patients had LC at AIH diagnosis $(p=0.12)$. None of the IgG4-AIH patients revealed any abnormal findings in the pancreas and bile ducts suggestive of IgG4-related diseases (IgG4$\mathrm{RD})$ at the diagnosis and during the study period.

In terms of liver histology, the IgG4-AIH patients revealed a significantly severer phenotype than the classical$\mathrm{AIH}$ patients in portal inflammation $(2.8 \pm 0.6$ [mean \pm $\mathrm{SD}$ ] vs. $2.2 \pm 0.8, p=0.018)$, interface hepatitis $(3.2 \pm 0.6$ vs. $2.2 \pm 1.2, p=0.01)$, fibrosis $(3.9 \pm 1.2$ vs. $2.5 \pm 1.4, p=$ $0.004)$, and rosette formation ( $1.5 \pm 0.7$ vs. $0.9 \pm 0.7, p=$ 0.026 ) (Fig. 3). There were no significant differences in plasma cell infiltration ( $1.9 \pm 0.8$ vs. $1.5 \pm 0.7, p=0.594)$, confluent necrosis $(2.6 \pm 1.4$ vs. $2.0 \pm 1.6, p=0.242)$, steatosis $(0.5 \pm 0.7$ vs. $0.6 \pm 0.8, p=0.664)$, and bile duct damage ( $0.3 \pm 0.5$ vs. $0.2 \pm 0.4, p=0.731)$ (Fig. 3$)$. The number

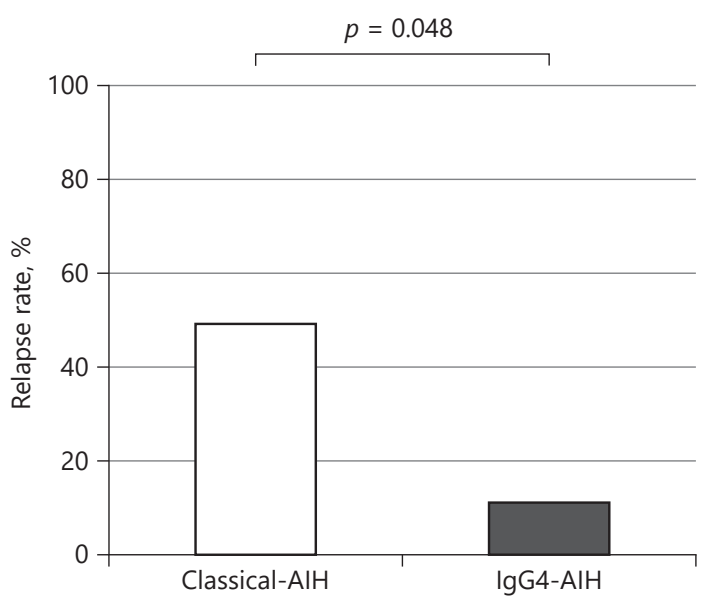

Fig. 4. Comparison of the relapse rate between IgG4-AIH and classical-AIH. The relapse rate was significantly lower in the IgG4$\mathrm{AIH}$ group than in the classical-AIH group (11.1 vs. $49.2 \%$; $p=$ 0.048). Ig, immunoglobulin; IgG4-AIH, IgG4-associated autoimmune hepatitis.

of IgG-positive plasma cells was significantly greater in the IgG4-AIH patients than the classical-AIH patients $(40.3 \pm 19.4$ [mean $\pm \mathrm{SD}$ ] vs. $17.5 \pm 18.7$ cells/HPF, $p=$ $0.005)$.

\section{Clinical Course of IgG4- and Classical-AIH}

Seventy-two patients $(85.7 \%)$ were initially treated with PSL, and the remaining 12 patients (14.3\%) were 


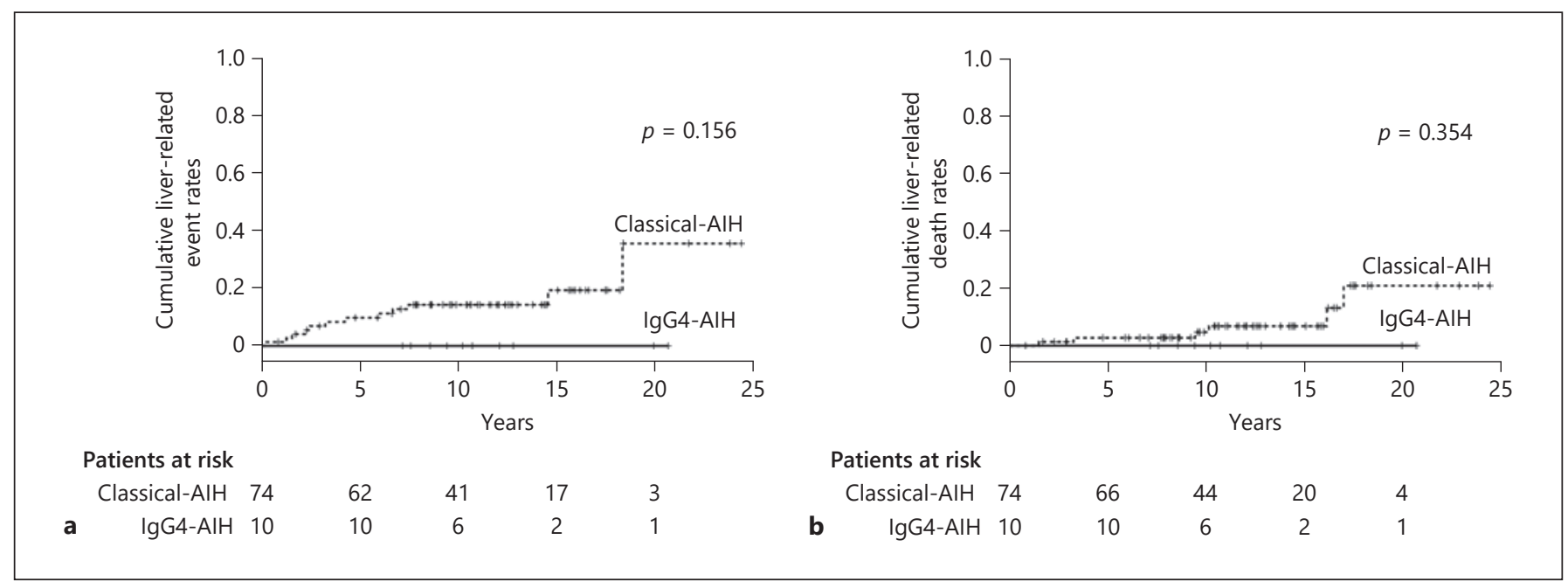

Fig. 5. a Cumulative liver-related event rates in patients with IgG4AIH (solid line) and classical-AIH (broken line). The cumulative rates of liver-related events were higher in the classical-AIH group than in the IgG4-AIH group, but the difference was not statistically significant $(p=0.156)$. b Cumulative liver-related death rates

started with a UDCA (600 mg/day) monotherapy. During a median follow-up of 139 (range: 10-292) months, we compared the clinical course and prognosis between IgG4-AIH and classical-AIH patients. Nine IgG4-AIH patients $(90.0 \%)$ and 63 classical-AIH patients $(85.1 \%)$ were treated with PSL at an initial dose of $20-60 \mathrm{mg} /$ day $(p=0.564)$ (Table 1), all of whom achieved biochemical remission followed by PSL maintenance therapy at a dose of $3-10 \mathrm{mg} /$ day. Of the 72 patients initially treated with PSL, relapse occurred in 32 patients (44.4\%) after ALT normalization. The relapse rate was significantly lower in the IgG4-AIH group than the classical-AIH group (11.1 vs. $49.2 \% ; p=0.048$ ) (Fig. 4). One classical-AIH patient initially treated with UDCA alone received PSL at a dose of $20 \mathrm{mg} /$ day due to continuous ALT elevation, and subsequently achieved remission. Four classical-AIH patients received azathioprine as an add-on therapy to PSL because of multiple relapses.

During the follow-up period, liver-related events occurred in 12 patients (14.3\%); development of esophageal varices, ascites, and HCC was observed in 6, 4, and 2 patients, respectively. All of the patients with liver-related events belonged to the classical-AIH group. The cumulative rates of liver-related events were higher in the classicalAIH group than the IgG4-AIH group, but the difference did not reach statistical significance ( $p=0.156$; Fig. 5a). Fifteen patients (17.9\%) died in this study. Of these, 6 , all of whom belonged to the classical-AIH group, died of liv- in patients with IgG4-AIH (solid line) and classical-AIH (broken line). The cumulative rates of liver-related death were higher in the classical-AIH than in the IgG4-AIH group, but the difference was not statistically significant $(p=0.354)$. Ig, immunoglobulin; IgG4$\mathrm{AIH}, \mathrm{IgG} 4$-associated autoimmune hepatitis.

er-related events; causes of 4 and 2 patients' death were liver failure and HCC, respectively. The cumulative rates of liver-related death were higher in the classical-AIH group than the IgG4-AIH group, but the difference was not statistically significant ( $p=0.354$; Fig. $5 b)$. Notably, none of the IgG4-AIH patients had liver-related events and death. The cumulative rates of liver-related events and death were not significantly different between the patients initially treated with PSL and those with UDCA alone.

\section{Discussion}

We showed that a certain proportion of AIH patients had IgG4-positive plasma cell infiltration in the liver; $11.9 \%$ of the AIH patients were diagnosed as IgG4-AIH according to our criteria. We also revealed that these patients had a lower relapse rate and good prognosis despite the existence of more active inflammation and advanced fibrosis in the liver than the classical-AIH patients.

Chronic inflammation due to IgG4-positive plasma cell infiltration was first reported in the pancreas $[13,14]$; however, it was later identified in a variety of organs including the bile duct, salivary glands, lungs, kidneys, intestinal tract, and retroperitoneum. As a result, a new disease concept, known as the IgG4-RD, was proposed [15, 16]. IgG4-positive plasma cell infiltration in the affected tissues is a common pathological feature of IgG4-RD. Umemura et al. [6] 
proposed a concept of IgG4-AIH, which is characterized by marked IgG4-positive plasma cell infiltration in the liver along with the elevation of serum IgG4 levels. His group reported that only $2(3.3 \%)$ of 60 Japanese patients with AIH fulfilled their criteria, which consisted of the number of IgG4-positive plasma cells $\geq 10 / \mathrm{HPF}$ and the serum IgG4 level $\geq 135 \mathrm{mg} / \mathrm{dL}$ [7]. On the other hand, Chung et al. [8] diagnosed 9 (34.6\%) of 26 Japanese AIH patients as IgG4AIH based on their criterion that the number of IgG4-positive plasma cells is $\geq 5$ cells/HPF. In Western countries, Canivet et al. [9] diagnosed 7 (25.0\%) of $28 \mathrm{AIH}$ patients as IgG4-AIH when they used the criterion of the number of IgG4-positive plasma cells $\geq 10 / \mathrm{HPF}$. IgG4 is a subtype of IgG, accounting for no more than $5 \%$ of total IgG under physiological conditions [17]. In the present study, we revealed a positive correlation between the numbers of IgG4and IgG-positive plasma cells in the liver tissue. In order to extract the patients who have IgG4-dominant liver infiltration, we set the IgG4/IgG ratio $\geq 5 \%$ as our diagnostic criteria. In addition, as actually no IgG4-positive plasma cells were observed in the majority of the cases $(63.1 \%)$, we considered the infiltration of IgG4-positive plasma cells $\geq 3$ / HPF in the portal area, which is the best cutoff value calculated from the ROC curve, to be significant. Therefore, IgG4-AIH was diagnosed when the patients fulfilled the following criteria: number of IgG4-positive plasma cells/ $\mathrm{HPF} \geq 3$ and $\mathrm{IgG} 4 / \mathrm{IgG}$ ratio $\geq 5 \%$ in this study. Using this criterion, 10 of the $84 \mathrm{AIH}$ patients were diagnosed as IgG4-AIH.

The IgG4-AIH patients had significantly more advanced portal inflammation, interface hepatitis, fibrosis, and rosette formation at the time of diagnosis than the classical$\mathrm{AIH}$ patients. Two patients diagnosed as IgG4-AIH according to the criteria of Umemura et al. [7] revealed high hepatic inflammatory activity with bridging fibrosis, giant cell change, and rosette formation. Chung et al. [8] also reported that there was a significantly higher degree of portal inflammation and rosette formation in the IgG4-AIH patients than classical-AIH patients. These findings are consistent with our results.

Furthermore, we, for the first time, analyzed the longterm clinical course of IgG4-AIH in comparison with the classical-AIH. The liver-related events and deaths were not observed in the IgG4-AIH patients. This is in contrast to the classical-AIH: 16.2 and $8.1 \%$ of the patients had liverrelated events and died of liver failure or HCC, respectively. The frequency of concurrent autoimmune diseases was higher in the classical-AIH group than in the IgG4-AIH group, but the difference was not statistically significant. Concurrent autoimmune diseases such as Sjögren's syn-

AIH with IgG4-Positive Plasma Cell Infiltration drome, systemic lupus erythematosus, and autoimmune thyroiditis were managed primarily by the rheumatologist, and the controls were very good. However, the impact on liver-related prognosis cannot be completely denied. We also demonstrated that the IgG4-AIH relapsed at a significantly lower rate than the classical-AIH. Infrequent relapse would reduce the corticosteroid adverse events such as osteoporosis, glycometabolic disorder, and cataracts by decreasing unnecessary PSL doses. Multiple relapses were related to poorer prognosis in AIH patients [4]. Therefore, low relapse rates might result in the decrease of liver-related events and deaths in the IgG4-AIH patients.

In this study, all of the IgG4-AIH patients had serum IgG4 levels $\geq 135 \mathrm{mg} / \mathrm{dL}$. In contrast, only 1 of the 74 classical-AIH cases (1\%) had a serum IgG4 level $\geq 135 \mathrm{mg} / \mathrm{dL}$. This particular subject revealed no IgG4-positive plasma cell infiltration in the liver and did not undergo relapse or liver-related events during the course. Chung et al. [8] reported that the serum IgG4 levels in the IgG4-AIH patients did not increase similarly to that in other AIH patients: this result is different from ours. The serum IgG4 level, which rises in the majority of IgG4-RD patients [16], is being used as one of the diagnostic criteria of IgG4-RD. We found a significant positive correlation between serum IgG4 levels and the number of IgG4-positive plasma cells in the liver. Further study on the significance of serum IgG4 in the IgG4-AIH is necessary.

Recently, Nakanuma et al. [18] proposed a strict definition for IgG4-AIH. These criteria define "Definite IgG4$\mathrm{AIH}^{\prime}$ as those who fulfill all of the following elements: serum IgG4 concentration $\geq 135 \mathrm{mg} / \mathrm{dL}$, increased IgG4-positive cells in the liver tissue $\geq 10 / \mathrm{HPF}$, chronic hepatitis with zonal and bridging necrosis or broad collapse, and metachronous or synchronous association with other IgG4-RD. As these criteria contain the existence of IgG4-RD as a mandatory requirement, "Definite IgG4-AIH" may represent a hepatic phenotype of IgG4-RD rather than a subtype of AIH $[19,20]$. A considerable proportion of patients with type 1 autoimmune pancreatitis (AIP) have accompanying liver dysfunction [21]. These patients had IgG4-positive plasma cell infiltration in the portal region as well as liver cell damage such as portal inflammation, large bile duct damage, portal sclerosis, lobular hepatitis, and cholestasis; interface hepatitis was observed in $24 \%$ of the AIP patients [21]. In addition, half of the AIP patients were diagnosed as probable AIH according to the IAIHG scoring system [7]. There is still an argument whether "Definite IgG4-AIH" is a subtype of AIH or a hepatic phenotype of IgG4-RD [19]. Furthermore, the majority of IgG4-RD patients are male, and the gendered difference in the degree of IgG4-positive 
plasma cell infiltration is unknown. In the present study, no patients manifested IgG4-RD symptoms. Further study is necessary to address this issue.

As the ability of IgG4 to activate complements is lower than that of other IgG subtypes [17], the pathogenicity of IgG4 in IgG4-RD is still unclear. Recently, Shiokawa et al. [22] subcutaneously injected IgG1 and IgG4 extracted from the blood of IgG4-RD patients into neonatal mice, which resulted in pancreatic and salivary gland injuries. IgG1 induced more destructive changes than IgG4. Interestingly, IgG1-induced injury was attenuated by the simultaneous IgG4 injection. These findings suggest that IgG1, but not IgG4, is the pathogenic culprit in IgG4-RD and that IgG4 functions as an anti-inflammatory agent. It remains unknown how IgG4 acts in IgG4-AIH patients.

There were several limitations in this study. First, the small number of patients analyzed in this study makes it difficult to draw a conclusion on the characteristics of IgG4-AIH. The occurrence rates of liver-related events and death in the IgG4-AIH were low compared with those in the classical-AIH patients, but the difference did not reach significance. Analysis of a larger cohort study may bring a meaningful result. Second, there is no consensus definition of IgG4-AIH. Utilizing our own criteria, we could discriminate IgG4-AIH from the classical-AIH in terms of histological features and relapse rates. Therefore, we believe that our criteria would be appropriate. Third, the number of patients who underwent the serum IgG4 tests was insufficient. Large-scale studies are necessary in the future.

\section{Conclusion}

We showed that IgG4-AIH revealed a low relapse rate and good prognosis despite higher degrees of inflammation and fibrosis in the liver tissue. Our findings suggest that IgG4-AIH may have a phenotype that differs from the classical-AIH.

\section{Acknowledgements}

We thank Noboru Kawabe at the Support Center for Medical Research and Education, Tokai University for technical assistance. We would like to thank Editage (http://www.editage.jp) for English language editing.

\section{Statement of Ethics}

The protocol of this study was approved by the Institutional Review Board for Clinical Research of Tokai University School of Medicine (No. 13R-120). All procedures were performed in accordance with the ethical standards of the institutional research committee andwith the 1964 Helsinki Declaration and its later amendments or comparable ethical standards. It was judged that written informed consent was not required in the Institutional Review Board for Clinical Research of Tokai University School of Medicine (no. 13R-120) because this research was a retrospective observational study and did not use genetic testing or additional samples from patients. Informed consent was obtained from all the participants by the opt-out method of the poster.

\section{Conflict of Interest Statement}

The authors have no conflicts of interest to declare.

\section{Funding Sources}

There was no financial support for this work.

\section{Author Contributions}

Arase Y., Nakazawa T., and Kagawa T. designed the study. Matsumoto K. performed the pathological evaluation. Arase Y., Anzai K., Tsuruya K., Sugiyama S., Yoshihara S., Hirose S., and Uojima H. collected and analyzed the data. Hidaka H., Nakazawa T., Deguchi R., Kojima S., Takashimizu S., Shiraishi K., and Shirai T. offered technical support. Arase Y. drafted the manuscript. All authors discussed and approved the final manuscript.

\section{References}

1 Krawitt EL. Autoimmune hepatitis. N Engl J Med. 2006 Jan 5;354(1):54-66.

2 Czaja AJ, Manns MP. Advances in the diagnosis, pathogenesis, and management of autoimmune hepatitis. Gastroenterology. 2010 Jul;139(1):58-e4.

3 Alvarez F, Berg PA, Bianchi FB, Bianchi L, Burroughs AK, Cancado EL, et al. International autoimmune hepatitis group report: review of criteria for diagnosis of autoimmune hepatitis. J Hepatol. 1999 Nov;31(5):929-38.
4 Yoshizawa $\mathrm{K}$, Matsumoto A, Ichijo $\mathrm{T}$, Umemura T, Joshita S, Komatsu M, et al. Long-term outcome of Japanese patients with type 1 autoimmune hepatitis. Hepatology. 2012 Aug;56(2):668-76.

5 Miyake Y, Iwasaki Y, Terada R, Takagi S, Okamaoto R, Ikeda H, et al. Persistent normalization of serum alanine aminotransferase levels improves the prognosis of type 1 autoimmune hepatitis. J Hepatol. 2005 Dec;43(6): 951-7.
6 Umemura T, Zen Y, Hamano H, Ichijo T, Kawa S, Nakanuma Y, et al. IgG4 associated autoimmune hepatitis: a differential diagnosis for classical autoimmune hepatitis. Gut. 2007 Oct;56(10):1471-2.

7 Umemura T, Zen Y, Hamano H, Joshita S, Ichijo T, Yoshizawa K, et al. Clinical significance of immunoglobulin G4-associated autoimmune hepatitis. J Gastroenterol. 2011 Jan;46(Suppl 1):48-55 
8 Chung H, Watanabe T, Kudo M, Maenishi O, Wakatsuki Y, Chiba T. Identification and characterization of IgG4-associated autoimmune hepatitis. Liver Int. 2010 Feb;30(2): 222-31.

9 Canivet CM, Anty R, Patouraux S, Saint-Paul MC, Lebeaupin C, Gual P, et al. Immunoglobulin G4-associated autoimmune hepatitis may be found in Western countries. Dig Liver Dis. 2016 Mar;48(3):302-8.

10 Aydemir Y, Akcoren Z, Demir H, Saltik Temizel IN, Ozen H, Yuce A. Clinical and histopathological features of immunoglobulin G4associated autoimmune hepatitis in children. J Gastroenterol Hepatol. 2019 Apr;34(4):7426.

11 Onji M, Zeniya M, Yamamoto K, Tsubouchi $\mathrm{H}$. Autoimmune hepatitis: diagnosis and treatment guide in Japan. Hepatol Res. 2014 Apr;44(4):368-70.

12 Ishak K, Baptista A, Bianchi L, Callea F, De Groote J, Gudat F, et al. Histological grading and staging of chronic hepatitis. J Hepatol. 1995 Jun;22(6):696-9.
13 Hamano H, Kawa S, Horiuchi A, Unno H, Furuya $\mathrm{N}$, Akamatsu $\mathrm{T}$, et al. High serum IgG4 concentrations in patients with sclerosing pancreatitis. N Engl J Med. 2001 Mar 8; 344(10):732-8.

14 Hamano H, Kawa S, Ochi Y, Unno H, Shiba $\mathrm{N}$, Wajiki M, et al. Hydronephrosis associated with retroperitoneal fibrosis and sclerosing pancreatitis. Lancet. 2002 Apr 20;359(9315): 1403-4.

15 Kamisawa T, Funata N, Hayashi Y, Eishi Y, Koike M, Tsuruta K, et al. A new clinicopathological entity of IgG4-related autoimmune disease. J Gastroenterol. 2003;38(10):982-4.

16 Stone JH, Zen Y, Deshpande V. IgG4-related disease. N Engl J Med. 2012 Feb 9;366(6):539_ 51.

17 Aalberse RC, Stapel SO, Schuurman J, Rispens T. Immunoglobulin G4: an odd antibody. Clin Exp Allergy. 2009 Apr;39(4):469-77.
18 Nakanuma Y, Ishizu Y, Zen Y, Harada K, Umemura T. Histopathology of IgG4-related autoimmune hepatitis and IgG4-related hepatopathy in IgG4-related disease. Semin Liver Dis. 2016 Aug;36(3):229-41.

19 Minaga K, Watanabe T, Chung H, Kudo M. Autoimmune hepatitis and IgG4-related disease. World J Gastroenterol. 2019 May 21; 25(19):2308-14.

20 Yada N, Kudo M, Chung H, Watanabe T. Autoimmune hepatitis and immunoglobulin G4-associated autoimmune hepatitis. Dig Dis. 2013;31(5-6):415-20.

21 Umemura T, Zen Y, Hamano H, Kawa S, Nakanuma Y, Kiyosawa K. Immunoglobulin G4-hepatopathy: association of immunoglobulin G4-bearing plasma cells in liver with autoimmune pancreatitis. Hepatology. 2007 Aug;46(2):463-71.

22 Shiokawa M, Kodama Y, Kuriyama K, Yoshimura K, Tomono T, Morita T, et al. Pathogenicity of IgG in patients with IgG4-related disease. Gut. 2016 Aug;65(8):1322-32. 